\title{
To the question about trust in the modern school
}

\author{
Tatyana Skripkina ${ }^{1,2, *}$, Elvira Vandanova ${ }^{1}$ \\ ${ }^{1}$ Russian State Humanitarian University 125993 Moscow, Russia \\ ${ }^{2}$ RANEPA, FIRO, 125319 Moscow, Russia
}

\begin{abstract}
The article gives a brief analysis of the monitoring, which was devoted to the study of the peculiarities of reflection of teachers about the presence of trust towards various entities of education in educational practice. Analysis of the obtained results has allowed to reveal features of trust in contemporary schools "through the eyes of teachers." Was also identified factors hindering and contributing to the development of trust between teachers, and subjects of educational activity: students, parents, administration.
\end{abstract}

\section{Introduction}

Despite the huge number of studies on various problems of education and educational activities, the problem of confidence in the school and confidence in school between subjects of educational activity does not lose its relevance and is still far from its full understanding [1]. Foreign sociologists and psychologists have called education the most important resource of "social capital" of society and of particular importance it is the trust factor [2].

In this regard, particular importance are the issues related to finding ways to address the problem, which goes deep into the category of psychological safety of the child at school. The importance of trust issues due to the fact that trust is the basis of the effectiveness of any joint activities or any of jointness [3]. However, the modern school has a huge number of problems associated with bullingham, alienation, rejection, cruelty, humiliation and many other negative phenomena, which have opposite nature [4]. The phenomenon of trust is a complex phenomenon, because confidence cannot be taught, it is impossible, no where invest to, no one can be forced or legally compelled to trust. The trust can only "grow" in the human soul, he is delegating the trust with responsibility for the executed instruction.

\section{Sample Description}

\footnotetext{
*Corresponding author: skripkinaurao@mail.ru
} 
Researchers have shown that in a holistic system - a person, there are two types of trust - this is self-confidence (reliance) on oneself in different areas of life and trust in others [5]. Based on this idea, the staff of the FIRO RANEPA conducted a large-scale monitoring dedicated to the study of trust and trust in educational practice "through the eyes of a teacher". Monitoring took place in on line mode. The total number of respondents was 18594 school teachers from all districts of the country.

\section{The study of the severity of self-confidence}

In order to assess the confidence of teachers in the medium and long term, a study was conducted in which teachers with different work experience took part. The hypothesis was as follows: the dynamics of differences or similarities in self-confidence among teachers with different work experience can serve as a means of forecasting trusting relationships in the educational environment. In the short term - this is the trust in teachers currently working in the school (including teachers with an experience of 20 years and above), in the medium term - trust in teachers with an experience of 5-20 years (based on the assumption that they will work to your retirement age), in the long term - the trust of experienced teachers (teachers with 1-5 years of experience).

During the analysis of the monitoring data, three groups of participants were identified, depending on work experience, half of the participants were experienced specialists, working retired teachers, one third were workers with seniority, and less than a quarter were young specialists.

\section{The study of the severity of trust in the subjects of educational activity}

The researchers developed two types of questions, one set of questions suggested choosing a ready-made answer and determining a measure of self-confidence in various areas of educational practice, the other - giving your answer and its justification on the subject of trust in other subjects of educational activity.

In the proposed questionnaire, in the first part of the study, they noted a measure of self-confidence: I fully trust, rather trust, partially trust, do not trust.

An analysis of the data on all the questions suggested showed that at the forefront of trust is a group of teachers with experience from 5 to 20 years. They will form the trust space of both the medium-term for the next 3 years and the long-term - the next 20 years.

In addition to questions related to self-confidence, teachers were asked several questions to which they had to give detailed answers. These questions were related to the reflection of trust in other subjects of educational activity. Just note that the detailed answers were not given by all teachers. Through content analysis, the detailed answers of the teachers were analyzed, and the number of similar answers to the selected semantic units was calculated. As a result, the following data were obtained.

Question: "Is personal human contact with students necessary?". As the data showed, the vast majority of teachers surveyed believe that personal contact with students is absolutely necessary. And, only $2.7 \%$ of the teachers who participated in the survey do not consider personal contact mandatory. However, they often write that their main task is to provide knowledge, rather than enter into relationships with students 
$13 \%$ of teachers who believe that personal contact is necessary is very pragmatic, as they believe that personal contact is the key to successful learning, helps the assimilation of information, etc. In fact, this part of teachers is inclined to use the presence of personal contact only in order to improve performance in school. The latter means that this group of teachers (and this is 13\%) is essentially not concerned with building a human trusting relationship, but basically reduces its professional activity only to the educational process.

Building up personal contacts with students in order to understand, support, help, and take into account individual characteristics, notes only $12.1 \%$ of the teachers surveyed. Thus, of all the teachers surveyed, despite the fact that the absolute majority indicates the need for establishing personal contact, only $25 \%$ substantiated the positions expressed by them. The rest of the teachers surveyed either did not substantiate their positions, or wrote about different aspects of the educational process, most often noted - to facilitate interaction with students, to establish a favorable climate in the classroom, to make it easier to influence the student, etc. So, the data obtained show that the vast majority of teachers believe that students need personal contact, but only $12.1 \%$ adequately reflect what it is for.

Question: "What prevents me from trusting students?". Most of the teachers, almost half, believe that nothing prevents them from trusting students - this is $48.7 \%$. We can assume that they have a confidential contact with students. Of the other answers, the most frequently encountered are claims to the character and personal qualities of students (arrogance, insincerity, conceit, irresponsibility, etc.) $-14.5 \%$ of the total number of respondents. A significant number of teachers note the falsehood and insincerity of students. This quality was noted by such a significant number of teachers $(12 \%)$ that we identified this attribute as a separate semantic unit.

It is important that the lack of trust in teachers on the part of students was noted by more than $5 \%$ of teachers who believe that students should trust them, not taking into account that trust is built by the teacher, and not the student. $4.6 \%$ of teachers believe that students do not need to be trusted, because, firstly, it reduces the status distance between them and students, secondly, this is impossible due to age differences, and thirdly, some say that they disappointed in trust in children and from experience shows. That students cannot be trusted. This group of teachers, as a rule, noted that children should respect elders. Another group of teachers stood out who were prevented from trusting their children by their irresponsible attitude to learning (3.5\%).

A significant part of teachers believe that parents prevent them from trusting parents, who either incorrectly raise their children, or inadequately relate to the teacher, or negatively set students in relation to teachers, etc. such answers turned out to be $4.5 \%$ of the entire sample

The remaining respondents gave the most varied answers - they called using gadgets, the Internet, student behavior, age differences, etc.

So, almost half of the educated teachers noted that the negative qualities of students, attitudes of parents, status distance, and an irresponsible attitude to learning prevented them from establishing confidential contact with students.

Question: "What prevents parents from trusting?". As the survey showed, almost $70 \%$ of teachers tend to trust their parents, however, at the same time, factors that impede the establishment of trusting relationships with students' parents were identified. These factors include: the insincerity of parents, sometimes their deceit, the desire to shield their child, to justify him, incorrect from the point of view of teachers, educational attitudes of parents, lack or lack of communication with parents, difference in values and age differences. The answer "aggression, disrespect, consumer attitude 
towards teachers, generated by the assumption that education is a service sector $(11 \%$ of respondents) occupies a special place in teachers' answers.

Question: "What prevents the administration from trusting?". Most of the respondents answered positively to this $-80.6 \%$ of teachers. Description of the pattern trust in the administration is contained in the statements: "I completely trust the administration", "I don't see any reason for discontent", "the administration is a school that came from our midst, part of our team and we completely trust them."

At the same time, the answers of the rest of the teachers suggest that such a large number of positive answers are socially desirable. In fact, teachers (of course not all) are afraid to give honest and sincere answers to this question, since, based on the answers, there is a feeling that in some cases the arbitrariness of the administration gives rise not only to distrust, but also the fear of sincerely answering this question. Teachers are afraid of sanctions. To confirm this assumption, we have analyzed in detail the answers of the remaining part of teachers - and this is 3032 people or $19.4 \%$. 17 patterns of relationship with the administration were compiled (along with a pattern of trust).

The bias pattern contains such statements about what disturbs trust: "lack of objectivity and a sense of tact (correctness) on the part of the school's administration", "biased attitude", "double standards".

The pattern is a lack of objective information - statements that interfere with trust: "untimely reporting", "discrepancies in the presentation of information", "secrecy and concealment of information".

The pattern of lies and deceit is "sometimes the administration is dishonest, there is no openness, it does not always make concessions", "deceit, empty promises from the administration."

The pattern of lack of stability is "uncertainty about tomorrow", "the teacher is not protected", "the possibility of dismissal", "non -transparency of the payment system". Teachers add "... I believe that there are no laws in education protecting the professional honor and human dignity of a teacher."

Alarmed by a large number of teachers who refused to answer this question. We can also highlight the fact that teachers feel unprotected: they can be fired, punished, reduced the payment of their incentive allowance, or even deprived of it. In the general sample, a small number of teachers indicated this, the percentage seems not to be large, relative to the entire sample, but this is almost 1000 people.

At first glance, the obtained results compel us to ask a question: Who are you teachers - professional cyborgs? Without a doubt, without reproaches, trusting themselves in everything. Or, for all socially desirable answers, we miss something important. As it turned out in this "sea" of trust, there are also pitfalls.

We list several problems described here, but also not included in this article, but obtained by analyzing the monitoring results.

First, some teachers believe that trust is not necessary at all in his professional activity, and this cannot be the competence of the teacher, his main task is to carry out educational activities. How should the educational process be built? Definitely not the way it is given here.

The second is aggression, disrespect, a consumer attitude to the teacher and school, the attitude of a certain part of parents to teachers as to attendants. How to work with a teacher when a parent is a destabilizing factor? In such a situation, teachers respond: "Not all the advice of parents is reasonable and applicable. It's bad when parents begin to realize their ambitions through children."

Third - there are cases when the administration focuses only on its own interests, what should teachers do - look for another job? Heads in education should understand 
that the participants in the organizational process are different: teachers, children, parents, men, women, and all of them require and deserve equal organizational and managerial support.

Fourth - the focus of management on reports, the so-called "paper files". It must be admitted that this method of leadership can give good results. But at a short distance. When it is possible to report to the higher authorities about successful results - now. Strategically, this is a way to destroy confidence.

The fifth is the amount of wages. We'll give one answer: "Wages are too low, although all the media say and write that teachers earn good money, incentive payments are allocated to the school once a quarter, and not monthly, and their size is ridiculous." It is difficult for a teacher to have an open conversation with management and administration. So, we need mechanisms that will compensate for low incomes on the one hand, and emotional losses (emotional burnout among teachers associated with professional activities) on the other.

\section{Conclusions and Conclusion}

So, the survey made it possible to identify in a generalized form some features of trusting relationships in a modern school with the "eyes of teachers".

\section{These features include:}

1. Most teachers absolutely trust themselves in the professional field (71\%). There is practically no teacher who considers himself a bad teacher.

2. Almost the same number of teachers fully trust themselves in relations with students $(71 \%)$. Only teachers with little work experience are not completely confident in their relationships with students.

3. In the ability to build relationships with parents, teachers trust themselves to a much lesser extent All teachers, regardless of work experience, can provide a confidential space for the educational environment. (58\%), of these teachers a little more than half.

\section{Confidence in relations with students:}

- Some teachers believe that trust, establishing personal contact with students is necessary for mutual understanding, assistance and support to the student;

- some teachers believe that trust is necessary as a factor that enhances the effectiveness of educational activities;

- some teachers believe that trust is not necessary at all in his professional activity, and this cannot be the competence of the teacher, his main task is to carry out educational activities.

Approximately half of teachers believe that they have confidential contact with students.

Factors that hinder the establishment of confidential contact with students:

- negative personal qualities of students, such as deceit, impudence, insincerity, irresponsibility, etc.

- irresponsible attitude to study, educational settings of parents.

- age differences and status relations between teachers and students, therefore, a distance is necessary in relations with students.

- Pupils should trust teachers, and not vice versa.

Factors that contribute to improving student relationships. 
Almost half of the teachers are completely satisfied with their relationships with students. What teachers would like to change in their relations with students:

- increase the demands on students in terms of discipline, behavior and respect for the teacher;

- have more trust, mutual understanding, openness;

- communicate more with students and spend more extra-curricular time with them;

- increase the demands on learning.

Confidence in relationships with parents.

More than half of teachers believe that they have a trusting relationship with parents. The rest identified factors that impede the establishment of trusting relationships with parents:

- aggression, disrespect, consumer attitude to the teacher and school;

- insincerity, a lie of parents in interaction with a teacher;

- lack of communication and information between the teacher and parents;

- the difference in values and age differences, different views on parenting;

- Features of parenting in the family;

- low cultural level of parents.

Factors making it difficult to trust the school administration.

It should be noted that the vast majority of teachers surveyed trust the administration. At the same time, approximately every fifth teacher (19.4\%) indicates the presence of factors that impede trust in the administration. Denote these factors in descending order:

- passivity, lack of support, statusless soulless position, mutual distrust, pursuit (on the part of the administration) of one's interests, different views and goals of lies, duplicity, indifference, indifference, bias in relation to, "double standards, disinterest in the employee:“ There are no irreplaceable people Say "thank you" for giving us money - personal qualities, interpersonal relationships, "human factor";

- formalism, weak positive motivation of teachers, bureaucratization, paperwork, lack of stability, insecurity in the future, insecurity, the possibility of dismissal, nontransparency of the payment system;

- life experience, negative experience of communication, negative experience of previous interactions, irrational behavior of those who are higher in office, unwillingness to understand the situation and help them deal with it, template thinking of the administration, linking managers to non-deterministic educational patterns;

- I am at a loss to answer, I do not know (put a dash).

\section{References}

1. T.P. Skripkina, Proceedings "From its origins to современности 130 years of the organization of the psychological society at the Moscow state University. M. V.

Lomonosov." 310-312 (2015)

2. P. Bourdieu, Structures, habitus, practices (Novosibirsk, 1995)

3. A.G. Asmolov, N.A. Pasternak, Child in adult culture (Moscow, 2019)

4. P.O. Omarova, Modern psychological and pedagogical researches of a problem of school violence (Penza, 2013)

5. T.P. Skripkina, Psychology of trust (Moscow, 2000) 